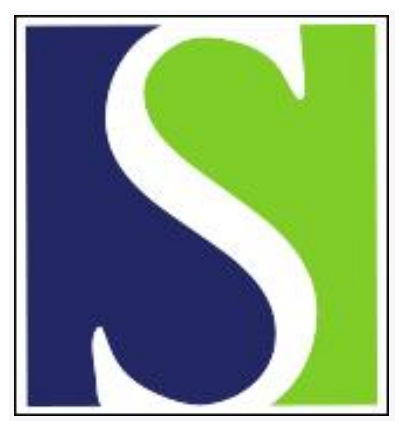

Scand J Work Environ Health 2002;28(4):215-221

https://doi.org/10.5271/sjweh.668

Issue date: Aug 2002

Effect of mental and physical demands on muscular activity during the use of a computer mouse and a keyboard

by Laursen B, Jensen BR, Garde AH, Jørgensen AH

Affiliation: National Institute of Public Health, Svanemøllevej 25, DK-2100 Copenhagen Ø, Denmark. bla@niph.dk

The following articles refer to this text: 2002;28(4):211-213;

2003;29(6):452-460; 2004;30(5):399-409; 2008;34(5):374-380

Key terms: computer mouse; electromyographic gap; electromyography; forearm; keyboard; mental demands; muscular activity; performance; physical demands; repetitive work; static muscle activity

This article in PubMed: www.ncbi.nlm.nih.gov/pubmed/12199422 


\title{
Effect of mental and physical demands on muscular activity during the use of a computer mouse and a keyboard
}

\author{
by Bjarne Laursen, PhD, ${ }^{1}$ Bente Rona Jensen, PhD, ${ }^{2}$ Anne Helene Garde, PhD, ${ }^{3}$ \\ Anker Helms Jørgensen, $P h D^{4}$
}

\begin{abstract}
Laursen B, Jensen BR, Garde AH, Jørgensen AH. Effect of mental and physical demands on muscular activity during the use of a computer mouse and a keyboard. Scand J Work Environ Health 2002;28(4):215-221.
\end{abstract}

\begin{abstract}
Objectives This study explores the effects of mental and physical demands on muscular activity in the use of the mouse and keyboard in computer work in a time-pressed situation.

Methods Twelve female subjects performed a computer task with four combinations of two forms of input device (computer mouse and keyboard) and two levels of mental demand (with and without a color word test). The time available for response was limited. Performance and electromyography (EMG) were recorded for the forearm, shoulder, and neck muscles.

Results The mean static muscular activity was 2-7\% of the maximum EMG for the forearm and neck extensor muscles. All the muscles for which recordings were made showed increased muscular activity in the mentally demanding condition. The increase in the static muscular activity level varied from $0.3 \%$ of the maximum EMG for the right trapezius muscle to $2.6 \%$ for the extensor carpi ulnaris muscle. Significant differences in muscular activity between the use of a computer mouse and a keyboard were only found for the neck extensors; in the computer mouse condition there was a static EMG level of 3.5\% for the maximum EMG, compared with $2.8 \%$ for the keyboard.

Conclusions Mental demands during computer work increased muscular activity in all of the recorded muscles. Increased muscular activity was found in the neck during the use of the mouse in comparison with the use of the keyboard; this phenomenon may be related to higher visual demands during the use of a mouse than with a keyboard. These findings may help explain the adverse effects of psychosocial work factors on the musculoskeletal system.
\end{abstract}

Key terms electromyography, electromyographic gaps, forearm, performance, repetitive work, static muscle activity.

A relation between the use of a visual display unit (VDU) and an increased prevalence of musculoskeletal symptoms has been documented in epidemiologic studies, for example, in those by Marcus \& Gerr (1), Bernard et al (2), and Yu \& Wong (3), and a review has been published by Punnett \& Bergqvist (4). Several potential risk factors have been recognized, for instance, physical factors such as poor workplace design and postures $(3,5)$ and psychosocial work factors $(1)$ such as time pressure due to deadlines (6). In most cases, VDU work includes the use of both a computer mouse and a keyboard. Although the use of the mouse has increased significantly during the last decade, little is known regarding the impact of its use on the musculoskeletal system. However, some studies indicate increased musculoskeletal symptoms in relation to mouse use $(7,8)$.

There is still a lack of knowledge as to the extent to which the use of different input devices influences the activation of the different muscles in the upper limbs. Besides the mechanical differences between the computer mouse and keyboard, the demands for hand-eye coordination differ. For trained typists, keyboard use

1 National Institute of Public Health, Copenhagen, Denmark.

2 Institute of Exercise and Sports Sciences, University of Copenhagen, Copenhagen, Denmark.

3 National Institute of Occupational Health, Department of Physiology, Copenhagen, Denmark.

4 The IT-University of Copenhagen, Copenhagen, Denmark.

Reprint requests to: Mr Bjarne Laursen, National Institute of Public Health, Svanemøllevej 25, DK-2100 Copenhagen $\emptyset$, Denmark. [E-mail: bla@niph.dk] 
requires no hand-eye coordination and, therefore, may result in a highly automated process with a typical typing speed of about 250 characters per minute (9). In contrast, the use of a computer mouse requires positioning of the mouse, and the relation between the mouse position and the screen cursor position is usually not absolute one-to-one correspondence. Therefore, computer mouse use requires extensive hand-eye coordination (10) and may therefore be more difficult to automate. These differences in motor demands between the character-based user interface (keyboard) and a graphic user interface (computer mouse) may be reflected in the workload. In addition to the motor demands imposed by the input device, computer tasks often comprise high mental demands. These demands may differ greatly, ranging from cognitive demands, to attention demands, to time pressure, and others. Mental demands have been found to increase the activity of some muscles, especially that of the trapezius muscle $(11,12)$. It was therefore hypothesized that, during computer work, motor demands and mental demands may interact and result in increased muscular activity.

The aim of this experiment was therefore to explore the effects of the mental and physical demands involved in computer work using the mouse and keyboard on muscular activity in a time-pressed situation.

\section{Subjects and methods}

\section{Subjects}

Twelve healthy female subjects without musculoskeletal symptoms in their forearms, shoulders, or neck gave their informed consent and participated in this laboratory study, as approved by the local ethics committee. The subjects were right-handed, and their mean age was 32 (range 27-41) years. All the subjects were experienced computer users and had experience using a computer mouse.

\section{Workplace design}

During the experiment, the subjects sat on an office chair, which was adjusted for the subject with respect to height, back support, and distance to the table. The table was adjusted to allow for forearm support. The mouse mat and the keyboard were positioned away from the body at a distance the length of the forearm. The subjects were allowed to move the mouse mat and the keyboard to the left and right. The screen (17", Sony CPD-200EST, resolution $1024 \times 768$ ) was kept free of reflections, and the top of the screen was aligned with the subject's eyes. After adjustments, the relative posi- tions of the chair, table, screen, mouse mat, and keyboard were fixed. The computer mouse was an ordinary symmetric mouse (Hewlett-Packard M-S34). The mouse sensitivity (screen cursor movement relative to mouse movement) was set at medium. This setting corresponded to six times larger movement on the screen when compared with the mouse movement when the mouse was moved slowly, and the movement was about 12 times larger when the mouse was moved quickly. All the computer work was carried out with the right hand.

\section{Tasks}

A computer task was performed with different combinations of input devices and mental demands. The input devices were the computer mouse (M) and the keyboard $(\mathrm{K})$, and mental demand was provided in the form of the use (Cwt) or nonuse (used as reference, Ref) of a computer version of the Stroop color word test. The four combinations of input device and mental demand resulted in the tasks MRef, KRef, MCwt, and KCwt. Each task lasted 8 minutes, followed by a break of 2 minutes. With each input device, the 8-minute $\mathrm{Cwt}$ period was repeated five times while different measurements were taken. The results presented for the Cwt condition are average values of the second and third period. The Ref task was performed for one 8-minute period with each input device. The task sequence was KRef, $5 \times \mathrm{KCwt}$, lunch break, $5 \times \mathrm{MCwt}$, MRef for six of the subjects and MRef, $5 \times \mathrm{MCwt}$, lunch break, $5 \times \mathrm{KCwt}$, KRef for the other six in balanced order. A training session preceded the tasks, and the lunchbreak was 2 hours.

The color word test served as a mental stress model $(13,14)$. In the task, two competing visual stimuli were presented. One of the Danish words for "red", "green", "blue", and "yellow" was shown on the computer screen in random order and at different positions on the screen. These color words were approximately 15 millimeters high, in Helvetica font, and on a gray background. The letters appeared in a color different from the color spelled by the word. Below the color word were four icons $(7 \times 11 \mathrm{~mm})$ labeled with the color words in black, always in the same order. The task for the subjects was to report the color of the word. The color words were presented at random intervals at a machine-determined pace for an average of 1.3 seconds (range 0.6-2.0 s). The subjects were instructed to enter the color of the word, either by clicking the icon with the correct answer using the computer mouse (mouse condition) or by hitting one of four adjacent keys, which corresponded to the icons on the screen (keyboard condition). The subjects were to answer as quickly and correctly as possible. An auditory alarm (beep) sounded when the subject gave a wrong answer or did not respond within the given time. For the $\mathrm{KCwt}$ and $\mathrm{MCwt}$ 
tasks, the percentages of correct answers relative to the number of presented color words were calculated.

In the MRef task, the subjects clicked a single icon, which appeared on the screen at the same positions and time intervals as for the MCwt task. In the KRef task, the subjects were instructed to press any of the four keys used in the KCwt task when an asterisk appeared on the screen. The asterisk appeared at the same positions and time intervals as the color word. In this way, the physical work demands were the same in the Cwt and Ref conditions.

\section{Maximum voluntary contractions}

Before the tasks, isometric maximum voluntary contractions (MVC) were performed. For wrist extension, the MVC were performed in a seated position with the elbow flexed 95 degrees and the forearm horizontal in the sagittal plane, pronated and supported, with the force transducer placed perpendicular to, and at the distal end of, the metacarpal bones. Finger extension MVC was performed with the force transducer placed perpendicular to the body of the phalanx. Ulnar deviation MVC was performed without a force recording. Maximum handgrip contraction was performed with the unsupported forearm in a neutral (thumb up) horizontal position. Maximum shoulder elevations were performed in a standing position with braces on both acromions. Maximum neck extension was performed with resistance against the fingers, which were interlaced behind the head; no force was measured. Each MVC was repeated at least three times. Up to two extra MVC were performed if the last MVC force was the largest. The EMG background level was measured while the subject was lying down.

\section{Electromyography and performance recording}

Electromyography (EMG) was recorded from the arm, shoulder, and neck muscles using bipolar $\mathrm{Ag}-\mathrm{AgCl}$ surface electrodes (N-10-A, Medicotest, Ølstykke, Denmark) with a distance of 20 millimeters between the recording areas. The muscles recorded in the right arm were the extensor carpi radialis (ExCarpRad), flexor carpi radialis (FlCarpRad), extensor digitorum (ExDig), and extensor carpi ulnaris (ExCarpUln). In the shoulder-neck area, the upper part of the trapezius muscles on the right and left side (Trap-R \& Trap-L, respectively) and the neck extensor muscle group on the right side (Neck) were recorded. The raw EMG was filtered $(2-450 \mathrm{~Hz})$, sampled $(1024 \mathrm{~Hz})$, and analyzed off-line. For each task, the root mean square (rms) of the EMG using intervals of 100 milliseconds, was calculated for 400 seconds, excluding the first 60 seconds and last 20 seconds of each task. All the EMG values obtained during the computer tasks were normalized to the maximum 1-second average EMG $\left(\mathrm{EMG}_{\max }\right)$ measured during the MVC. The static muscular activity (the 10-percentile level, ie, the level exceeded $90 \%$ of the time) and the mean level were determined for each task. Furthermore, the rate of the EMG gaps and the relative time with EMG gaps were calculated. The EMG gaps were defined as periods with EMG of less than $0.5 \%$ of the $\mathrm{EMG}_{\max }$ for at least 0.2 seconds (15), the rate of the EMG gaps was the number of EMG gaps per minute, and the relative time with EMG gaps was the percentage of time in which EMG gaps occurred.

Time curves were determined for the muscle activation of each muscle as the average curve for all responses (keystrokes or mouse clicks) during one of the 8minute periods for each of the four tasks (KRef, MRef, $\mathrm{KCwt}, \mathrm{MCwt})$. The curves were aligned relative to the keystroke or mouse click (time 0), which was checked to be within 0.01 seconds.

\section{Statistics}

Two-way repeated measurements in an analysis of variance (ANOVA) were used for testing the effect of the input devices ( $\mathrm{M}$ and $\mathrm{K}$ condition) and the effect of mental demand (Cwt and Ref conditions), and subjects were treated as a random factor. The EMG for each muscle was tested separately, and the Wilcoxon signed rank test was used for testing the differences between the muscles.

\section{Results}

\section{Performance}

The subjects performed significantly better with the keyboard than with the mouse, the mean of the percentage of correct answers during the color word test being 82 (SEM 3)\% with the keyboard and 54 (SEM 4)\% with the mouse. The mean response time for the correct answer was 0.70 (SEM 0.02) seconds with the KCwt task and 1.09 (SEM 0.02) seconds with MCwt task. Without mental demand, the response time (11 subjects) was 0.45 (SEM 0.02) seconds for the KRef task and 0.89 (SEM 0.02) seconds for the MRef task. All the differences in response time were significant (KRef $<\mathrm{KCwt}<\mathrm{MRef}<\mathrm{MCwt}$ ).

\section{Electromyography}

The static and mean EMG levels are presented in table 1 for the four tasks. The ANOVA showed a significant effect for input device with respect to the neck extensors 
Table 1. Static and mean electromyographic (EMG) value as the percentage of the maximum EMG (\%EMG ${ }_{\max }$ ) for the four tasks and $P$ values for the main effects. (ExCarpRad = extensor carpi radialis muscle, ExDig = extensor digitorum muscle, ExCarpUIn = extensor carpi ulnaris muscle, FICarpRad = flexor carpi radialis muscle, Trap $-\mathrm{R}=$ right trapezius muscle, Trap $-\mathrm{L}=$ left trapezius muscle, Ref $=$ reference task, $\mathrm{Cwt}=$ color word test, $\mathrm{M}=$ mouse, $\mathrm{K}=$ keybord, $\mathrm{SE}=$ standard error of the mean)

\begin{tabular}{|c|c|c|c|c|c|c|c|c|c|c|c|c|c|c|}
\hline & \multicolumn{12}{|c|}{$E M G\left(\% E M G_{\max }\right)$} & & \\
\hline & \multicolumn{2}{|c|}{ ExCarpRad } & \multicolumn{2}{|l|}{ ExDig } & \multicolumn{2}{|c|}{ ExCarpUIn } & \multicolumn{2}{|c|}{ FICarpRad } & \multicolumn{2}{|l|}{ Trap-R } & \multicolumn{2}{|l|}{ Trap-L } & \multicolumn{2}{|l|}{ Neck } \\
\hline & Static SE & Mean SE & Static SE & Mean SE & Static SE & Mean SE & Static SE & Mean SE & Static SE & Mean SE & Static SE & Mean SE & Static SE & Mean SE \\
\hline \multicolumn{15}{|l|}{ Mouse } \\
\hline Ref & 2.10 .3 & 3.20 .4 & 6.30 .6 & 9.50 .9 & 3.50 .5 & 6.40 .8 & 2.00 .4 & 7.01 .7 & $\begin{array}{ll}0.4 & 0.2\end{array}$ & $\begin{array}{ll}0.8 & 0.2\end{array}$ & 0.20 .1 & $\begin{array}{ll}0.7 & 0.1\end{array}$ & 3.50 .5 & 4.80 .6 \\
\hline Cwt & 2.60 .4 & 3.90 .5 & 7.10 .7 & 10.21 .1 & 4.00 .5 & 7.50 .9 & 2.90 .6 & 8.02 .0 & 0.90 .3 & $\begin{array}{ll}1.5 & 0.4\end{array}$ & 0.70 .1 & 1.30 .2 & $4.5 \quad 0.6$ & $\begin{array}{ll}6.0 & 0.7\end{array}$ \\
\hline \multicolumn{15}{|l|}{ Keyboard } \\
\hline Ref & 2.00 .4 & 3.30 .6 & 6.00 .7 & 10.20 .9 & 3.50 .6 & 6.10 .8 & 1.10 .2 & 2.70 .3 & $0.3 \quad 0.2$ & 0.80 .3 & 0.10 .0 & $\begin{array}{ll}0.7 & 0.2\end{array}$ & 2.80 .5 & 4.20 .6 \\
\hline Cwt & 2.50 .4 & 3.90 .7 & $\begin{array}{ll}6.8 & 0.7\end{array}$ & 11.41 .0 & 6.10 .7 & 9.10 .9 & 1.90 .3 & 4.50 .6 & $0.6 \quad 0.2$ & $\begin{array}{ll}1.2 & 0.3\end{array}$ & 0.70 .2 & 1.30 .3 & $3.6 \quad 0.5$ & $\begin{array}{ll}5.3 & 0.7\end{array}$ \\
\hline \multicolumn{15}{|l|}{ P-value a } \\
\hline Ref vs Cwt & 0.006 & 0.004 & 0.033 & 0.038 & $<0.001$ & $<0.001$ & $<0.001$ & 0.004 & 0.009 & 0.015 & 0.003 & 0.002 & $<0.001$ & $<0.001$ \\
\hline $\mathrm{M}$ vs K & 0.70 & 0.95 & 0.69 & 0.39 & 0.089 & 0.41 & 0.098 & 0.052 & 0.43 & 0.60 & 0.70 & 0.93 & 0.004 & 0.004 \\
\hline
\end{tabular}

a P-values are based on $F(1,11)$ from the analysis of variance test.

Table 2. Electromyographic (EMG) gaps in the four tasks. ${ }^{a}$ (ExCarpRad $=$ extensor carpi radialis muscle, ExDig $=$ extensor digitorum muscle, ExCarpUIn = extensor carpi ulnaris muscle, FICarpRad = flexor carpi radialis muscle, Trap-R = right trapezius muscle, Trap- $\mathrm{L}=$ left trapezius muscle, $\mathrm{Ref}=$ reference task, $\mathrm{Cwt}=$ color word test, $\mathrm{SE}=$ standard error of the mean)

\begin{tabular}{|c|c|c|c|c|c|c|c|c|c|c|c|c|c|c|}
\hline & \multicolumn{14}{|c|}{ EMG gaps/minute } \\
\hline & \multicolumn{2}{|c|}{ ExCarpRad } & \multicolumn{2}{|l|}{ ExDig } & \multicolumn{2}{|c|}{ ExCarpUln } & \multicolumn{2}{|c|}{ FICarpRad } & \multicolumn{2}{|c|}{ Trap-R } & \multicolumn{2}{|c|}{ Trap-La } & \multicolumn{2}{|l|}{ Neck } \\
\hline & Mean & SE & Mean & SE & Mean & SE & Mean & SE & Mean & SE & Mean & SE & Mean & SE \\
\hline \multicolumn{15}{|l|}{ Mouse } \\
\hline Ref & 0.0 & 0.0 & 0.0 & 0.0 & 0.2 & 0.2 & 1.0 & 0.6 & 16.3 & 5.5 & 49.8 & 7.6 & 0.2 & 0.1 \\
\hline Cwt & 0.0 & 0.0 & 0.0 & 0.0 & 0.0 & 0.0 & 0.0 & 0.0 & 12.9 & 5.2 & 23.3 & 7.6 & 0.0 & 0.0 \\
\hline \multicolumn{15}{|c|}{ Keyboard } \\
\hline Ref & 2.7 & 1.8 & 0.2 & 0.2 & 2.7 & 2.5 & 8.2 & 5.1 & 16.8 & 6.4 & 61.2 & 7.9 & 3.0 & 2.0 \\
\hline Cwt & 0.0 & 0.0 & 0.0 & 0.0 & 0.1 & 0.1 & 1.6 & 1.5 & 12.0 & 4.9 & 35.7 & 9.0 & 0.8 & 0.8 \\
\hline
\end{tabular}

a Significant difference between the color word test and the reference task.

with a higher mean and static activity when the mouse was used and, furthermore, a tendency towards a difference between input devices for the mean EMG for the FlCarpRad muscle. For the neck extensors, the static activity was 3.5\% during the MRef task, compared with $2.8 \%$ of the $\mathrm{EMG}_{\max }$ during the KRef task. The Cwt condition caused higher mean and static muscular activity in all the muscles, compared with the Ref condition. For the static EMG component, the increase in muscular activity due to the Cwt condition compared with the Ref condition varied from $0.3 \%$ of the $\mathrm{EMG}_{\max }$ for the Trap-R muscle with the use of the keyboard to $2.6 \%$ of the $\mathrm{EMG}_{\max }$ for the ExCarpUln muscle with the use of the keyboard. A comparison of the mean of the four tasks showed that the ExDig muscle had the largest mean EMG and the Trap-R and Trap-L muscles had the least, in comparison with the other muscles (Wilcoxon test).
The numbers of EMG gaps per minute are presented in table 2, and the relative time with EMG gaps are presented for the four different tasks in figure 1. Significant differences between the Cwt and Ref tasks were found for the relative time with EMG gaps for the Trap$\mathrm{R}$ and Trap- $\mathrm{L}$ muscles $(\mathrm{P}<0.01)$ and in the gap rate for Trap-L; the least gaps were found for the Cwt condition. For the remaining muscles, almost no gaps were found. There was a tendency towards a smaller relative time with EMG gaps when the mouse was used $(\mathrm{P}=0.069$ for the geometric mean of relative time with EMG gaps for the seven muscles), but none for the muscles separately.

The time courses of the EMG activities are shown for the forearm muscles in figure 2. EMG peaks were found close to the clicks for all the forearm muscles. Especially for the FlCarpRad muscle, a higher EMG peak was found in the $\mathrm{K}$ condition. For the shoulder and 
neck muscles there were no systematic variations in the EMG activity.

\section{Discussion}

In this study, the addition of mental demands during computer work caused an increase in the muscular activity of all the muscles for which values were recorded. Previous studies have shown an effect of mental demands on the trapezius muscle $(11,12,16)$ and the wrist extensor muscles $(11,17)$ but not on the wrist flexor muscles (11). Our study showed a similar effect of mental demands on all the muscles in the forearm, shoulder, and neck. This effect was observed as an increase in muscular activity, both during and between the clicks. There may be several explanations for this increase. A direct, centrally mediated physiological effect of the mental workload induced by the color word test may be one contributing factor that may result in higher general muscular tone and lead to a higher static EMG level and fewer EMG gaps. Furthermore, the mental demands may have had an indirect effect on muscular activity because they made it more difficult to perform the task within the given time (ie, increased time pressure). Time pres- sure may result in more forceful and jerkier movements when demands for precision increase, as has been found in a previous study (18). For the Trap-L muscle and the neck, the same absolute increases were found for the mean and static EMG levels, the finding indicating primarily a centrally mediated effect of mental demand on muscular activity. Particularly for the FlCarpRad muscle with the use of the keyboard, the effect on the mean

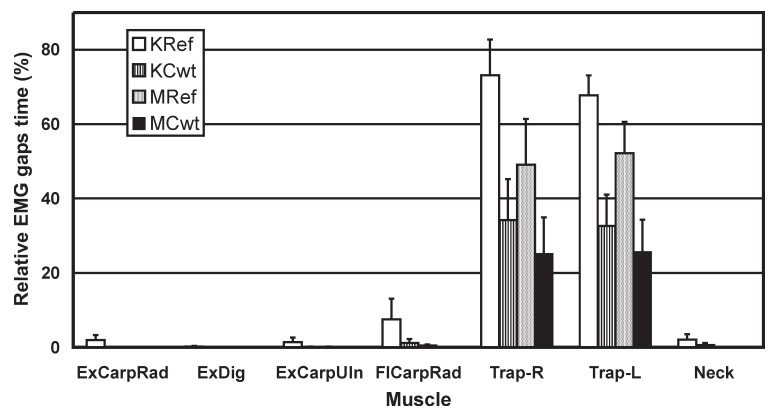

Figure 1. Relative time with electromyographic (EMG) gaps for the four tasks. The results are the means and the standard error of the means of 12 subjects. $(\mathrm{K}=$ keyboard, $\mathrm{M}=$ mouse, $\mathrm{Cwt}=$ color word test, Ref $=$ reference task, ExCarpRad $=$ extensor carpi radialis muscle, FICarpRad $=$ flexor carpi radialis muscle, ExDig = extensor digitorus muscle, ExCarpUln = extensor carpi ulnaris muscle)
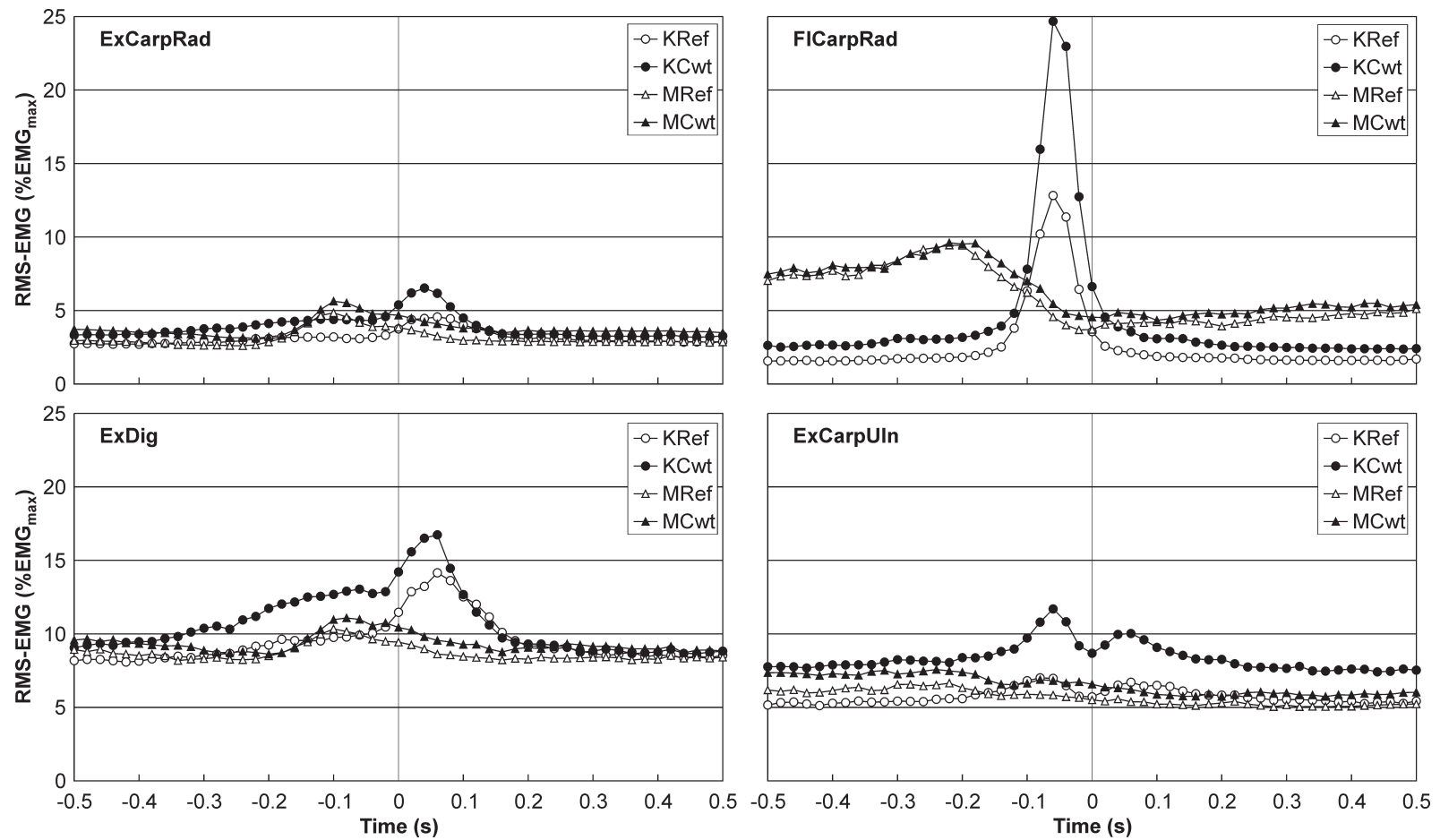

Figure 2. Time course of the electromyographic (EMG) activity in the forearm muscles during mouse and keyboard clicking, mean of 12 subjects. The four panels show the forearm muscles. (Time $=0$ when the computer detects the click, ExCarpRad $=$ extensor carpi radialis muscle, FICarpRad $=$ flexor carpi radialis muscle, ExDig = extensor digitorus muscle, ExCarpUln = extensor carpi ulnaris muscle, $\mathrm{K}=$ keyboard, $\mathrm{M}=\mathrm{mouse}, \mathrm{Cwt}=\mathrm{color}$ word test, Ref $=$ reference task, RMS = root mean square) 
EMG was stronger than that on the static EMG and thus indicated that the keys were pressed more forcefully during the mentally demanding tasks. This possibility is confirmed by the time course of the muscular activity, which showed a considerably higher EMG peak in association with key pressing in the Cwt tasks. The effect of mental demands towards a higher and more static load on the upper extremities may be one explanation for the adverse effects of psychosocial work factors on the musculoskeletal system.

In our study, a significant difference was found between use of the computer mouse and the keyboard for mean and static muscular activation in the neck extensor muscles, the highest EMG level being measured in the mouse condition. This difference may have been caused by the increased demands for hand-eye coordination with the use of the mouse, the increase leading to eye fixation on the screen - and probably to a need for shorter eye reaction time. Activation of neck extensor muscles reduces the reaction time of the eye (19), and the visual demands may therefore be a possible explanation for the increased muscular activity in the neck with the use of the mouse.

For the remaining muscles, no effect was found for the input device on mean and static muscle activation. This result corresponds to those found in other studies, which have shown no consistent differences between the two devices (20-22). However, in the present study, the time courses of the muscular activities differed for the mouse and the keyboard. No clear relation between muscle activation and clicking of the mouse button was observed, probably because almost no net force was needed.

A muscular activity pattern with almost no EMG gaps may be crucial, as it has been shown that few EMG gaps may be related to the development of work-related musculoskeletal symptoms (23). One of the mechanisms behind this relationship may be an association between EMG gaps and the rotation of motor unit recruitment (24), which thus possibly prevents the overload of muscle fibers. In our study, very few EMG gaps were found, and both input devices may increase the risk of symptoms in the forearm, the computer mouse possibly causing the largest effect. This finding is supported by a recent study showing continuous motor-unit activity during mouse clicking (25).

The EMG levels were particularly high for the ExDig muscle. The high level in the ExDig muscle for the use of both the keyboard and the mouse cannot be explained by external force demands. One likely explanation may be that the stability demands and time pressure of the task may have led to coactivation of the finger extensors and flexors so that fast clicking of either the keyboard or the mouse could be performed or the accidental pressing of keys could be avoided (26).
The task was performed considerably faster using the keyboard than using the mouse. This difference is probably due to the more complex hand-eye coordination needed when the mouse is used. This finding is reflected in the higher number of correct answers when the keyboard was used. The task of our study was chosen to represent one that could be solved using both a computer mouse and a keyboard, corresponding to the choice between clicking an icon with the mouse and using a shortcut key. However, other tasks will favor one of the input devices over the other. Therefore, the type of task may, to some extent, influence the results.

In conclusion, mental demands during computer work increase muscular activity in general, and this phenomenon indicates an increased central drive to the muscles, as well as a movement-related component due to motor demands. Increased muscular activity in the neck was found in the mouse condition when compared with the keyboard condition. This finding may be related to higher visual demands in association with the computer mouse than with the keyboard. For the other muscles, no differences between the two devices were found. This experiment shows a clear effect for mental demands with higher and more static muscular activity. This finding may help explain the adverse effects of psychosocial work factors on the musculoskeletal system.

\section{Acknowledgments}

This study was financially supported by the Danish Government.

\section{References}

1. Marcus M, Gerr F. Upper extremity musculoskeletal symptoms among female office workers: associations with video display terminal use and occupational psychosocial stressors. Am J Ind Med 1996;29:161-70.

2. Bernard B, Sauter S, Fine L, Petersen M, Hales T. Job task and psychosocial risk factors for work-related musculoskeletal disorders among newspaper employees. Scand J Work Environ Health 1994;20:417-26.

3. Yu ITS, Wong TW. Musculoskeletal problems among VDU workers in a Hong Kong bank. Occup Med 1996;46:275-80.

4. Punnett L, Bergqvist U. Visual display unit work and upper extremity musculoskeletal disorders: a review of epidemiological findings. Solna: National Institute for Working Life, 1997. National Institute for Working Life - ergonomic expert committee document, no 1.

5. Bergqvist U, Wolgast E, Nilsson B, Voss M. Musculoskeletal disorders among visual display terminal workers: individual, ergonomic, and work organizational factors. Ergonomics 1995;38:763-76. 
6. Polanyi MFD, Cole DC, Beaton DE, Chung J, Wells R, Abdolell M, et al. Upper limb work-related musculoskeletal disorders among newspaper employees: cross-sectional survey results. Am J Ind Med 1997;32:620-8.

7. Fogleman M, Brogmus G. Computer mouse use and cumulative trauma disorders of the upper extremities. Ergonomics 1995;38:2465-75.

8. Karlqvist L, Hagberg M, Köster M, Wenemark M, Ånell R. Musculoskeletal symptoms among computer-assisted design (CAD) operators and evaluation of a self-assessment questionnaire. Int J Occup Environ Health 1996;2:185-94.

9. Sommerich CM, Marras WS, Parnianpour M. Observations on the relationship between key strike force and typing speed. Am Ind Hyg Assoc J 1996;57:1109-14.

10. Ferrel C, Leifflen D, Orliaguet J-P, Coello Y. Pointing movement visually controlled through a video display: adaptation to scale change. Ergonomics 2000;43:461-73.

11. Wærsted M, Westgaard RH. Attention-related muscle activity in different body regions during VDU work with minimal physical activity. Ergonomics 1996;39:661-76.

12. Wærsted M, Bjørklund RA, Westgaard RH. The effect of motivation on shoulder-muscle tension in attention-demanding tasks. Ergonomics 1994;37:363-76.

13. Frankenhaeuser M, Johansson G. Task demand as reflected in catecholamine excretion and heart rate. J Hum Stress 1976;2:15-23

14. Hoshikawa Y, Yamamoto Y. Effects of Stroop color-word conflict test on the autonomic nervous system responses. Am J Physiol 1997;273:1113-21.

15. Veiersted KB, Westgaard RH, Andersen P. Pattern of muscle activity during stereotyped work and its relation to muscle pain. Int Arch Occup Environ Health 1990;62:31-41.

16. Larsson S-E, Larsson R, Zhang Q, Cai H, Öberg PÅ. Effects of psychophysiological stress on trapezius muscles, blood flow and electromyography during static load. Eur J Appl Physiol 1995;71:493-8.
17. Tulen JHM, Moleman P, van Steenis HG, Boomsma F. Characterization of stress reactions to the stroop color word test. Pharmacol Biochem Behav 1989;32:9-15.

18. Laursen B, Jensen BR, Sjøgaard G. Effect of speed and precision demands on human shoulder muscle electromyography during a repetitive task. Eur J Appl Physiol 1998;78: $544-8$.

19. Kunita K, Fujiwara K. Relationship between reaction time of eye movement and activity of the neck extensors. Eur J Appl Physiol 1996;74:553-7.

20. Jensen C, Finsen L, Hansen K, Christensen H. Upper trapezius muscle activity patterns during repetitive manual material handling and work with a computer mouse. J Electromyogr Kinesiol 1999;9:317-25.

21. Cooper A, Straker L. Mouse versus keyboard use: a comparison of shoulder muscle load. Int J Ind Ergon 1998;22:351-7.

22. Fernström E, Ericson MO. Computer mouse or tracpoint effects on muscular load and operator experience. Appl Ergon 1997;28:347-54.

23. Veiersted KB, Westgaard RH, Andersen P. Electromyographic evaluation of muscular work pattern as a predictor of trapezius myalgia. Scand J Work Environ Health 1993;19: 284-90.

24. Westgaard RH, De Luca CJ. Motor unit substitution in longduration contractions of the human trapezius muscle. J Neurophysiol 1999;82:501-4.

25. Søgaard K, Sjøgaard G, Finsen L, Olsen HB, Christensen H Motor unit activity during stereotyped finger tasks and computer mouse work. J Electromyogr Kinesiol 2001;11:197206.

26. Laursen B, Jensen BR, Ratkevicius A. Performance and muscle activity during computer mouse tasks in young and elderly adults. Eur J Appl Physiol 2001;84:329-36.

Received for publication: 9 October 2001 